\title{
La Comunicación de las Organizaciones no Lucrativas del ámbito de la Salud en España: aproximaciones a una matriz de análisis
}

\author{
Hernán Alfredo DíAz \\ Universidad Complutense de Madrid \\ hernandi@ucm.es \\ Ubaldo Cuesta CAmbra \\ Universidad Complutense de Madrid \\ ucuestac@ucm.es
}

\begin{abstract}
Resumen:
Las Organizaciones No Lucrativas (ONL) del ámbito de la salud tienen un protagonismo creciente. La comunicación tiene un carácter estratégico para la gestión de estas organizaciones, pero la investigación en este campo es todavía insuficiente. Los autores desarrollaron una matriz de análisis de la comunicación con aportaciones de Pérez (2008), Grunig (2011) y Villafañe (2013), que luego aplicaron en un estudio de casos de carácter empírico exploratorio sobre cinco ONL del ámbito de la salud de España. La matriz será aplicada en futuras investigaciones sobre una muestra más representativa.
\end{abstract}

Palabras clave: Gestión; Comunicación; Organizaciones No Lucrativas; Salud; Estrategias.

\section{Communication of Nonprofit Organizations in the field of Health in Spain: approaches to an analytical framework.}

\begin{abstract}
:
Nonprofit Organizations (NPOs) in the field of health have an increasing role. Communication is strategic for the management of these organizations, but research in this field is still insufficient. The authors developed a matrix of communication analysis with input from Perez (2008), Grunig (2011) and Villafane (2013), which was applied in a exploratory empirical study of five spanish NPOs in the field of health. The analytical framework will be applied in future research on a more representative sample.
\end{abstract}

Key Words: Management; Communication; Non Profit Organizations; Health; Strategies

\section{Referencia normalizada:}

Alfredo Díaz, H. y Cuesta Cambra, U. (2014): La comunicación de las organizaciones no lucrativas del ámbito de la salud en España: aproximaciones a una matriz de análisis. Historia y Comunicación Social. Vol. 19. Núm. Especial Enero. Págs. 209-219.

Sumario: 1. Introducción. 2. Marco conceptual y metodológico. 2.1. 2.1. Los conceptos que articulan la matriz de análisis. 2.2. Los métodos de la investigación empírica. 3. Algunas generalizaciones a partir de los estudios de caso. 3.1. Variables de estrategias. 3.2. Variables de estructura. 3.3. Variables de procesos. 3.4. Variables tácticas. 4. Acerca de la necesidad de una matriz de análisis. 5. Referencias bibliográficas. 


\section{Introducción}

El cruce de la comunicación y la salud se constituyó como campo de estudio en las últimas décadas del siglo XX. Como han señalado Ugarte (2008) y Terrón (2010), la investigación en comunicación y salud tiene una sólida raíz en los países anglosajones, que han sido los impulsores de este campo de estudio en torno al concepto de health communication. En cambio, en el ámbito iberoamericano el desarrollo es más reciente y todavía embrionario, al punto de que se carece todavía de un concepto único y se utilizan diferentes expresiones como "comunicación y salud" (Cuesta, 2008; Terrón, 2010; Petracci y Waisbord, 2011), "comunicación para la salud (Coe, 1998; Díaz, 2011) o "comunicación en salud" (Obregón, 2010; Muñoz-Cruzado, 2010). De todos ellos, el de "comunicación y salud" se ha ido imponiendo en el ámbito académico, en buena medida porque pone en pie de igualdad a esos dos ámbitos de estudio.

Una revisión sistemática de la literatura científica (Beck, 2004; Thompson, 2006; Freimuth, 2006; Kim, 2010; Nazione, 2013) muestra que la investigación en comunicación y salud se ha focalizado en dos grandes temas: la información de salud y las relaciones entre profesionales sanitarios y pacientes. El análisis de esa bibliografía indica que cuando se ha investigado sobre comunicación de organizaciones se lo hecho prioritariamente sobre aquellas que prestan servicios sanitarios asistenciales, como hospitales y centros de salud, tanto públicos como privados.

Este estado de la cuestión se contrapone con una realidad incontrastable: el creciente protagonismo de las Organizaciones No Lucrativas (ONL) en el ámbito de la salud. Según datos aportados por el Anuario del Tercer Sector de Acción Social de la Fundación Luis Vives (2012), en España actualmente hay alrededor de 29.000 organizaciones de este tipo, de las cuales el $22,1 \%$ tienen como campo de actuación principal el socio-sanitario, solo por detrás de la acción social $(38,6 \%)$ y la integración e inserción $(23,2 \%)$. Estamos hablando de más de 6.000 organizaciones activas, entre las que se encuentran asociaciones profesionales y de pacientes, fundaciones, plataformas y sociedades científicas.

El protagonismo alcanzado por las ONL en el ámbito de la salud tiene su raíz en la evolución del concepto mismo de salud, desde una concepción limitada a un fenómeno físico de "ausencia de enfermedad" hacia un enfoque bio-psico-social que la definió como "el estado de completo bienestar físico, psíquico y social" (OMS, 2006). Esa evolución conceptual hizo que la salud dejara de ser un tema de competencia exclusiva del sistema sanitario y trajo consigo nuevas enfoques y estrategias, que empezaron a poner el énfasis en el control de los determinantes sociales de la salud, especialmente en lo referido a los entornos físicos y sociales en los que viven las personas y a los hábitos y estilos de vida que adoptan (Lalonde, 1974; OMS, 1986; OMS, 2001; Missoni, 2012).

La comunicación tiene para las ONL un carácter estratégico porque se relaciona con su capacidad para dar visibilidad a sus acciones y construir su reputación; incor- 
porar determinada temática en la agenda pública; posicionarse como interlocutor social; construir redes con otras organizaciones; y dar cuenta a la sociedad de forma transparente de sus actuaciones (Vernis, 1998; Canel y Echart, 2005; Herranz de las Casas, 2007; Balas, 2012; Fundación Luis Vives; 2012). Sin embargo, pese a ese carácter estratégico, la comunicación de las ONL del ámbito de la salud sigue siendo un campo de investigación prácticamente inexplorado.

El análisis precedente señala la necesidad de desarrollar una línea de investigación que estudie la comunicación de las ONL del ámbito de la salud, en el marco de la cual se desarrollen instrumentos teóricos y prácticos para la gestión profesional de la comunicación en estas organizaciones.

\section{Marco conceptual y metodológico}

En línea con planteamientos que conciben a la comunicación en un sentido estratégico y relacional, en procesos de interacción y construcción social de sentido basados en el reconocimiento de los diferentes actores implicados en los procesos de salud y sus particularidades sociales y culturales (Coe, 1998; Cuesta, 2008; Díaz y Uranga, 2011; Díaz, 2011; Petracci y Waisbord, 2011), hemos desarrollado una matriz de análisis para abordar de forma integral la gestión de la comunicación de las ONL del ámbito de la salud.

\subsection{Los conceptos que articulan la matriz de análisis}

Esta matriz incorpora las aportaciones de la Nueva Teoría de la Estrategia (Pérez y Massoni, 2008), fundamentalmente en lo referido a la necesidad de desarrollar una visión sistémica de las organizaciones, así como los desarrollos conceptuales de Jaramillo (2011) y Pérez (2012) acerca de las diferencias entre "lo estratégico" y "las estrategias".

Asimismo, asumimos como perspectiva comunicacional la que ofrece el campo de las relaciones públicas. Destacan las aportaciones de Grunig $(1984 ; 2011)$; respecto de la importancia que tiene para una organización la construcción de relaciones simétricas, de colaboración y entendimiento mutuo con sus públicos estratégicos, en procesos que Wilcox et al. (2012) caracterizaron como sistemáticos, planificados e intencionados.

El tercer elemento fundamental de nuestra propuesta es la teoría reputacional, siguiendo los planteamientos de Villafañe $(2003 ; 2013)$ en el sentido de que la reputación es la consolidación de una buena imagen corporativa como fruto de un comportamiento excelente reconocido a lo largo del tiempo y de forma estructural por sus grupos de interés estratégicos.

Con fines explicativos y analíticos, la matriz se articula en cuatro categorías de variables -estrategias, estructura, procesos y tácticas-, aunque en la práctica los lími- 
tes entre una y otra categoría puedan ser más difusos y muchas de las variables son interdependientes:

- Variables estratégicas. Pretenden analizar si la comunicación forma parte de los ejes estratégicos de la organización, considerando también su integración y traslación a la concepción y los objetivos de comunicación.

- Variables estructurales. Aportan información relevante sobre la influencia que tiene la estructura y los actores responsables de la comunicación en la toma de las decisiones y su carácter estratégico o táctico.

- Variables de proceso. Analizan la sistematicidad de la gestión en las diferentes etapas de ese proceso (diagnóstico, planificación, ejecución y evaluación), así como las utilización de algún tipo de matriz de variables e indicadores que sirva como marco de referencia.

- Variables tácticas. Este apartado refiere a las actividades y productos a través de los cuales la gestión la comunicación de una organización toma cuerpo.

En la siguiente tabla incluimos todas las variables que forman parte de la matriz, integrando las aportaciones de la teoría estratégica, las relaciones públicas y la reputación descriptas previamente.

Tabla 1. Matriz de análisis de la gestión de la comunicación de las ONL del ámbito de la salud. Elaboración propia.

$\begin{array}{ll}\text { Categoría } & \text { Variables } \\ \text { Variables } & \text { V1. Planteamiento estratégico de visión, misión y valores } \\ \text { estratégicas } & \text { V2. Identificación de grupos de interés } \\ & \text { V3. Concepción de comunicación } \\ & \text { V4. Objetivos de comunicación } \\ \text { V5. Articulación entre "lo estratégico" y estrategias de comunicación } \\ \text { Variables } & \text { V6. Lugar en el organigrama } \\ \text { estructurales } & \text { V7. Denominación del área } \\ & \text { V8. Funciones asignadas } \\ & \text { V9. Carácter integral de la comunicación } \\ & \text { V10. Perfil de los gestores de la comunicación } \\ & \text { V11. Recursos financieros } \\ \text { Variables de proceso } & \text { V12. Diagnóstico comunicacional } \\ & \text { V13. Planificación de la comunicación } \\ & \text { V14. Ejecución de la comunicación } \\ \text { V15. Evaluación de la comunicación } & \text { V16. Productos de comunicación } \\ \text { Variables tácticas } & \text { V17. Comunicación en medios masivos } \\ & \text { V18. Eventos } \\ \text { V19. Comunicación 2.0 }\end{array}$




\subsection{Los métodos de la investigación empírica}

Una vez construida la matriz, se desarrolló una investigación empírica exploratoria, utilizando para ello dos técnicas cualitativas: el estudio de casos y las entrevistas en profundidad.

Como señalan Wimmer y Dominick (1996), la técnica del estudio de casos es un método muy útil para comprender o explicar un fenómeno, obtener detalles variados y hallar pistas o ideas preliminares para investigaciones posteriores. En nuestra investigación estudiamos cinco casos de ONL del ámbito de la salud: Fundación 1000 para la prevención de malformaciones congénitas; Esclerosis Múltiple España; Asociación Sexualidad y Discapacidad; Grupo Español de Pacientes con Cáncer (GEPAC); y Sociedad Española de Cardiología / Fundación Española del Corazón (SEC/FEC). Se trata de una muestra de organizaciones seleccionada a partir de criterios de diversidad (profesionales y pacientes; investigación, divulgación y promoción de la salud); tipologías (asociaciones, fundaciones y federaciones) y la accesibilidad a las mismas.

En el marco de los estudios de caso se realizaron entrevistas en profundidad a los responsables de comunicación de esas organizaciones, para conocer con el mayor detalle posible la gestión, su evolución y los modelos teóricos o analíticos que actúan como marco de referencia. Se elaboró un cuestionario base para las entrevistas, que recogía preguntas referidas a las cuatro categorías de variables recogidas en la matriz de análisis. Las entrevistas se realizaron a los responsables directos de la gestión de la comunicación: en las entidades que disponen de una área específica de comunicación, a la persona con mayor responsabilidad en la gestión de este área; en las que no disponen de ese área, a la persona que asume en la práctica esa gestión.

\section{Algunas generalizaciones a partir de los estudios de caso}

En este apartado nos detendremos en el análisis de los resultados obtenidos para cada una de las categorías de análisis. No haremos referencias particulares a los casos de las ONL del ámbito de la salud estudiadas, aunque incluiremos citas textuales de las personas entrevistadas para respaldar las generalizaciones que hemos extraído.

\subsection{Variables de estrategias}

El análisis de estos casos señala que hay una preeminencia de las estrategias de comunicación, enfocadas a proyectos o acciones coyunturales con una visión de corto plazo, por encima de "lo estratégico", entendido como las definiciones que determinan el camino a seguir por las organizaciones en el mediano y largo plazo.

Existe consenso en que la comunicación es una dimensión estratégica de las organizaciones. En ese marco, se considera que el principal objetivo es dar visibilidad 
pública a las acciones desarrolladas por las organizaciones, con un claro enfoque a los públicos externos:

La comunicación es fundamental porque se traduce en visibilidad, y esta a su vez se traduce en interés por parte de los pacientes y las personas afectadas.

Hacer que la gente nos conozca para tener cierta influencia. No es importante solo hacer bien las cosas, sino que se sepa. Y para conseguir recursos, si no tienes visibilidad es muy difícil.

Que lo que hacemos no parezca un secreto.

Se aprecian diferencias en los modelos de comunicación de las organizaciones de profesionales y de pacientes. Mientras que las de profesionales tienen una concepción de comunicación más vertical, encuadrada en el modelo de información pública, las organizaciones de pacientes apuestan por una comunicación bidireccional simétrica, en los términos planteados por Grunig y Hunt (1984).

La comunicación es esencial: comunicación científica y basada en pruebas científicas. Hacemos traslación de conocimientos científicos a las personas que deben utilizarlos: los profesionales sanitarios y población general. (Organización de profesionales)

Se supone que los pacientes son el centro del SNS, pero no se les tiene en cuenta a la hora de tomar decisiones. Intentamos que se conozcan sus necesidades, inquietudes y experiencias. (Organización de pacientes).

Interactuamos mucho con los pacientes y con otras organizaciones de pacientes. (Organización de pacientes).

\subsection{Variables de estructura}

Todas las organizaciones estudiadas atribuyen como función principal del área de comunicación la información y la relación con los medios masivos. Como aspecto diferencial, las organizaciones que tienen un área específica suelen tener una visión más compleja, dentro de una perspectiva de relación con sus diferentes públicos, no limitado a los medios masivos.

Cuando todas las funciones de comunicación están integradas en una sola área de gestión, suele tener un carácter más estratégico y sistemático:

El Departamento de Comunicación influye en todos los otros, pero a la vez sabe lo que hace todo el resto.

Coordinamos al menos lo elemental, tratando de dar mensaje común y coherente con el movimiento mundial.

En línea con los planteos de Grunig (2011), los estudios de casos sugieren que las organizaciones que tienen ubicada su área de comunicación en el primer nivel del organigrama (toma de decisiones) tienen una visión más estratégica y bidireccional, 
en contraste con aquellas que la tienen ubicada en un segundo nivel (instrumental), donde la comunicación adquiere un carácter más táctico y unidireccional.

\subsection{Variables de procesos}

Del estudio de los casos se desprende que las organizaciones que tienen un área específica de comunicación desarrollan procesos más metódicos, con una asignación de roles y tareas y una calendarización de actividades que da cierto carácter sistemático a la gestión. Sin embargo, la mayoría de las organizaciones estudiadas carece de un plan de comunicación anual y solo desarrollan planes específicos de campañas o eventos especiales (en general, para poder captar recursos).

Hace algunos años la comunicación era más improvisada, menos metódica. Según iban viniendo las cosas ibas haciendo. Falta de tiempo y menos experiencia. Ahora si no tengo un calendario y no lo veo, resulta mucho más caótico.

Cuando hay una acción nueva hay una planificación previa, que incluye a qué medios les puede interesar, quién nos puede apoyar (como personajes públicos), qué entidades pueden estar interesadas en estas iniciativas.

Hay una tendencia a incorporar a actores de otras áreas en la planificación de la comunicación desde una visión más sistémica de la organización, promoviendo espacios de discusión interna sobre estrategias y tácticas en la fase de diseño de los planes.

Nos sentamos prácticamente toda la oficina y hablamos: podríamos hacer esto, podríamos hacer lo otro... Y de ahí sacamos lema, objetivos, actividades que se van a hacer, página web... Entre todos se decide que se va a hacer y qué se va a contar, luego cada uno en su rama lo desarrolla más y lo presenta.

La investigación está prácticamente ausente en la gestión de la comunicación de las ONL del ámbito de la salud. Las organizaciones estudiadas no disponen de una matriz de análisis ni de variables o indicadores que sirvan para la gestión de la comunicación en las diferentes etapas del proceso (diagnóstico, planificación, ejecución y evaluación).

No tenemos variables de análisis explicitadas. Nos interesa todo, lo cualitativo y lo cuantitativo, pero no de una manera exhaustiva.

\subsection{Variables tácticas}

Las organizaciones estudiadas asumen la importancia de tener una actitud proactiva en la gestión de la comunicación, tanto en su relación con los medios masivos como en el uso de las redes sociales. Aquellas organizaciones que disponen de una estructura específica de comunicación tienen estrategias más complejas, utilizando los eventos como "perchas" informativas que les permiten tener visibilidad pública.

Atendemos a los medios que nos solicitan información y a veces nos ofrecemos a los medios cuando ha surgido una noticia, para ser parte de la noticia con nuestra opinión. 
Nuestra estrategia ha pasado de ser reactiva a ser proactiva, de pensar acciones comunicables. No que te lo den hecho y te digan: comunica esto.

Internet y las redes sociales ocupan un lugar cada vez más relevante dentro de la comunicación de todas las organizaciones, dispongan o no de un área específica:

Tienen mucha importancia para nosotros y cada vez forman más parte de nuestro trabajo cotidiano.

Las redes sociales permiten que seamos un medio de comunicación. Es un recurso súper útil, es un error no estar.

Somos muy activos en redes sociales, en las que podemos llevar.

\section{Acerca de la necesidad de una matriz de análisis}

La creciente profesionalización de los departamentos de comunicación en las ONL del ámbito de la salud sin duda ha permitido una mejora en la gestión, pero según nuestra investigación exploratoria sobre cinco estudios de caso muestran la ausencia de un modelo analítico de referencia para la gestión de la comunicación. Esta podría ser una de las causas (o la consecuencia) del predominio de la visión táctica sobre la estratégica que se observa en muchas organizaciones, cuestión que habrá que profundizar en estudios posteriores.

De la misma manera, hemos observado que la investigación está prácticamente ausente de los procesos de comunicación, circunscrita a la recogida de información sobre impactos en medios masivos $\mathrm{y}$, de forma incipiente, a análisis cualitativos asistemáticos de las interacciones en redes sociales. Por lo anterior, los procesos de comunicación de estas organizaciones pueden calificarse de parcialmente metódicos y metodológicamente asistemáticos.

En línea con lo planteado por Balas (2012) en su estudio sobre la comunicación del Tercer Sector, se observa que estas organizaciones carecen de planes anuales de comunicación, prevaleciendo una planificación táctica enfocada a desarrollar productos y acciones con una visión de corto plazo. Por lo tanto, un de los desafíos para estas organizaciones es poder desarrollar procesos de gestión de la comunicación con un carácter más estratégico, basados en una visión de medio y largo plazo.

Parafraseando a Kurt Lewin, estamos convencidos de que "no hay nada más práctico que una buena teoría". La matriz de análisis que hemos desarrollado en este trabajo se ha mostrado como un instrumento útil para el análisis de la comunicación de estas organizaciones, por lo que apostamos por avanzar en su validación con una muestra más representativa para poder identificar los matices propios de la gestión de la comunicación de estas organizaciones del ámbito de la salud. 


\section{Bibliografía}

BALAS, M. (2012). La gestión de la comunicación en el Tercer Sector. Madrid: ESIC Editorial.

BECK, C. et al. (2004). Enacting "Health Communication": The field of Health Communication as constructed Through Publication in Scholarly Journals. Health Communication, Vol. 16, $\mathrm{n}^{\circ}$ 2. p. 475-492.

CANEL, M.J.; ECHART, N. (2005). La comunicación de las Fundaciones. En Organización y funcionamiento de las Fundaciones. Asociación Española de Fundaciones. p. 61-84.

COE, G. (1998). Comunicación y Promoción de la Salud. Revista Latinoamericana de Comunicación Chasqui, $\mathrm{n}^{\circ}$ 63. p. 26-29.

CUESTA, U.; MENÉNDEZ, T.; UGARTE, A. (Coords) (2008). Comunicación y Salud. Avances en modelos y estrategias de intervención (pp. 19-38). Madrid: Editorial Complutense.

DÍAZ, H. (2011). La comunicación para la salud desde una perspectiva relacional. En CUESTA, U.; MENÉNDEZ, T.; UGARTE, A. (Coords), Comunicación y Salud. Nuevos escenarios y tendencias. Madrid: Editorial Complutense. p. 33-49.

DÍAZ, H.; URANGA, W. (2011). Comunicación para la salud en clave cultural y comunitaria. Revista de Comunicación y Salud, Vol. 1, no 1. p. 113-124.

FREIMUTH, V.; MASSETT, H.; MELTZER, W. (2006). A Descriptive Analysis of 10 Years of Research Published in the Journal of Health Communication. Journal of Health Communication, 11 (1), 11-21.

FUNDACIÓN LUIS VIVES (2012). Anuario del Tercer Sector de Acción Social en España. Madrid: Fondo Social Europeo.

GRUNIG, J.; HUNT, T. (1984). Dirección de relaciones públicas. Edición adaptada al castellano por Jordi Xifra. Barcelona: Ediciones Gestión 2000.

GRUNIG, J. (2011). Characteristics of Excellent Communication. En GILLIS, T. (Ed.), The IABC Handbook of Organizational Communication. San Francisco: International Association of Business Communicators.

HERRANZ DE LACASA, J.M.(2007). La gestión de la comunicación como elemento generador de transparencia en las organizaciones no lucrativas. CIRIEC-España, Revista de Economía Pública, Social y Cooperativa, no 57. p. 5-31.

JARAMILLO LÓPEZ, J. C. (2011). ¿Comunicación estratégica o estrategias de comunicación? El arte del ajedrecista. Documento presentado en el VII Simposio Latinoamericano de Comunicación Organizacional, Universidad Autónoma de Occidente, Cali, Colombia.

KIM, J.; PARK, S.; YOO, S.; SHEN, H. (2010). Mapping Health Communication Scholarship: Breadth, Depth, and Agenda of Published Research in Health Communication. Health Communication, 25:6-7. p. 487-503.

LALONDE, M. (1974). A new perspective on the Health of Canadians. Ottawa: Ministery of Health Care and Welfare of Canada. Descargado el 15 de junio de $2013 \mathrm{de}$ http://www.fundadeps.org/Observatorio.asp?codrecurso=132

MISSONI, E. (2012). Rol y contribución de las organizaciones no gubernamentales y de la sociedad civil en la promoción de la salud global. En JUVINYÀ, D.; 
ARroyo, H. (Eds), La Promoción de la Salud 25 años después. Publicaciones de la Cátedra de Promoción de la Salud de la Universidad de Girona. Girona: Documenta Universitaria. p. 253-271.

MUÑOZ-CRUZADO Y BARBA, M. (2010). La comunicación en salud. Revista Española de Comunicación en Salud, Vol. 1, número 2. p. 66-67

NAZIONE, S.; PACE, K.; RUSSELL, J.; SILK, K. (2013). A 10-Year Content Analysis of Original Research Articles in Health Communication and Journal of Health Communication (2000-2009). Journal of Health Communication, 18. p. 223-240. ORGANIZACIÓN MUNDIAL DE LA SALUD (1986). Carta de Ottawa para la Promoción de la Salud. Ginebra, OMS. Descargado el 20 de mayo de 2013 de http://www1.paho.org/spanish/hpp/ottawachartersp.pdf

ORGANIZACIÓN MUNDIAL DE LA SALUD (2001). Alianzas estratégicas. El papel de la sociedad civil en el ámbito de la salud. Documento de trabajo, 1, 2001, CSI/2001/DP1. Ginebra: OMS. Descargado el 20 de mayo de 2013 de http://www. who.int/civilsociety/documents/en/AlianzasEstrategicas2001-DP1-sp.pdf

ORGANIZACIÓN MUNDIAL DE LA SALUD (2006). Constitución de la OMS. Documentos básicos. Descargado el 20 de mayo de 2013 de http://www.who.int/ governance/eb/who_constitution_sp.pdf

OBREGÓN, R. (2010). Un panorama de la investigación, teoría y práctica de la comunicación en salud. Revista Folios, no 23. p. 13-29.

PÉREZ, R. A.; MASSONI, S. (2008). Hacia una teoría general de la estrategia. Barcelona: Ariel.

PÉREZ, R.A. (2012). 2011: el año en que la comunicación se volvió estratégica. Revista DIRCOM, nº 93. p. 43-49.

PETRACCI, M.; WAISBORD, S. (2011) (Coords.). Comunicación y salud en la Argentina. Buenos Aires: La Crujía.

TERRÓN, J.L. (2010). Algunas reflexiones sobre la comunicación y salud en España tras cinco años del Observatorio de Comunicación y Salud. Revista Española de Comunicación en Salud, Vol. 1, n². p. 77-97.

UGARTE, A. (2008). El binomio comunicación y salud: relaciones e investigación. En CUESTA, U.; MENÉNDEZ, T.; UGARTE, A. (Coords), Comunicación y Salud. Avances en modelos y estrategias de intervención. Madrid: Editorial Complutense. p. 39-47.

VERNIS, A. el al. (1998). La Gestión de las Organizaciones No Lucrativas. Bilbao: Deusto.

VILLAFAÑE, J. (2003). La buena reputación. Claves del valor intangible de las empresas. Madrid: Pirámide.

Villafañe, J. (2013) La buena empresa. Propuesta para una teoría de la reputación corporativa. Madrid: Pearson Educación.

WILCOX, D.; CAMERON, G.; XIFRA, J. (2012). Relaciones públicas. Estrategias y tácticas. Madrid: Pearson Educación.

WIMMER, R.; DOMINICK, J. (1996). La investigación científica de los medios de comunicación. Una introducción a sus métodos. Barcelona: Bosch Casa Editorial. 


\section{Los autores}

Hernán Alfredo Díaz Máster en Comunicación de las Organizaciones por la Universidad Complutense de Madrid. Coordinador académico del Magíster en Comunicación y Salud (UCM).

Ubaldo Cuesta Cambra Doctor en Psicología. Catedrático de Psicología Social de la Comunicación y Director de la Cátedra Extraordinaria de Comunicación y Salud (Universidad Complutense de Madrid, España). 